\title{
Medical robotics simulation framework for application-specific optimal kinematics
}

Sven Böttger, Institute for Robotics and Cognitive Systems, University of Lübeck, Lübeck, Germany, boettger@rob.uni-luebeck.de

Tolga-Can Çallar, Institute for Robotics and Cognitive Systems, University of Lübeck, Lübeck, Germany, tolgacan.callar@student.uni-luebeck.de

Achim Schweikard, Institute for Robotics and Cognitive Systems, University of Lübeck, Lübeck, Germany, schweikard@ rob.uni-luebeck.de

Elmar Rückert, Institute for Robotics and Cognitive Systems, University of Lübeck, Lübeck, Germany, rueckert@ rob.uni-luebeck.de

Most kinematic structures in robot architectures for medical tasks are not optimal. Further, the workspace and payloads are often oversized which results high product prices that are not suitable for a clinical technology transfer. To investigate optimal kinematic structures and configurations, we have developed an adaptive simulation framework with an associated workflow for requirement analyses, modeling and simulation of specific robot kinematics. The framework is used to build simple and cost effective medical robot designs and was evaluated in a tool manipulation task where medical instruments had to be precisely positioned and oriented on the patient's body. The model quality is measured based on the maximum workspace coverage according to a configurable scoring metric. The metric generalizes among different human body shapes that are based on anthropometric data from UMTRI Human Shape. This dexterity measure is used to analyze different kinematic structures in simulations using the open source simulation tool V-REP. For that we developed simulation and visualization procedures for medical tasks based on a patchwork of size-variant anatomical target regions that can be configured and selectively activated in a motion planning controller. In our evaluations we compared the dexterity scores of a commercial lightweight robot arm with 7 joints to optimized kinematic structures with 6, 7 and 8 joints. Compared to the standard hardware, we achieved improvements of 59 percent when using a 6dimensional robot arm, 64\% with the 7-dimensional arm and 96\% with an 8-dimensional robot arm. Our results show that simpler robot designs can outperform the typically used commercial robot arms in medical applications where the maximum workspace coverage is essential. Our framework will be improved in order to provide a fully automatic optimization tool of the robot parameters and can be applied to a large variety of problems. 


\section{IEEE 11073 Compliant SDC Modelling of a Collaborative Robot for the Application of Focused Ultrasound in Radiation Therapy}

Johann Berger, Innovation Center Computer Assisted Surgery (ICCAS), Leipzig, Germany, johann.berger@medizin.uni-leipzig.de

Michael Unger, Innovation Center Computer Assisted Surgery (ICCAS), Leipzig, Germany, michael.unger@ medizin.uni-leipzig.de

Lisa Landgraf, Innovation Center Computer Assisted Surgery (ICCAS), Leipzig, Germany, lisa.landgraf@ medizin.unileipzig.de

Andreas Melzer, Innovation Center Computer Assisted Surgery (ICCAS), Leipzig, Germany, andreas.melzer@ medizin.uni-leipzig.de

The integration of surgical robotics into the operating room $(\mathrm{OR})$ requires reliable communication with other medical devices. In the OR.NET project, the IEEE 11073 SDC (Service-oriented Device Connectivity) standard was developed for dynamic device interconnectivity. Utilizing this standard, we have shown concepts and evaluations towards a combined focused ultrasound and radiation therapy (FUS-RT) to position interventional devices with collaborative KUKA arms. Ensuring safe and intraoperative cooperation of the robot in the OR implies a highly sophisticated exchange of the robot's information.

The aim of this work was the implementation and evaluation of an SDC compliant connection between collaborative KUKA robots and other medical devices. Therefore, the properties of a KUKA LBR iiwa 7 R800 robot (KUKA AG, Germany) were modeled with the SDC conform medical device description and evaluated on a mobile platform to position a Clarius L7 wireless ultrasound transducer as end effector of the robot arm (Clarius Mobile Health Corp, Canada). With this implementation, the position, torque, stiffness, damping, velocity, and functional-states of each robot-joint can be shared via network. The accessibility of the resulting 42 parameters was evaluated with an SDC-consumer application. The communication-software was written in $\mathrm{C}++$ and performed on a PC accessing the KUKA controller cabinet with ROS (Robot Operating System) via Ethernet.

The successful implementation of the robots SDC-provider functionality allows for dynamic changes of the robot state during interventions. All SDC standard compatible appliances in the robots network can react accordingly and perform movement commands via activations. Testing the 42 parameters resulted in accessibility of $100 \%$ by the SDCconsumer. This enables the KUKA robot to be integrated into any OR or radiation room network that supports the SDC standard and therefore to be installed and evaluated for the application of FUS-RT in a clinical environment. 


\section{Development and validation of 2D and 3D ultrasound thermometry algorithms}

Elina Werner, Marc Fournelle, Steffen Tretbar

Ultrasound Department, Fraunhofer IBMT, Sankt Ingbert, Germany

e-mail: steffen.tretbar@ibmt.fraunhofer

Ultrasound therapy involving HIFU (high intensity focused ultrasound) has become a non-invasive alternative to surgery for ablation of (malignant) tissue. By using array technology and multichannel electronics, ultrasound can be precisely focused to a ROI and thereby thermally ablate tissue in a confined volume. In clinical systems (e.g. Exablate/Insightec or Sonalleve/Philips), MR thermometry is used for monitoring therapeutic ultrasound, known as MR guided focused ultrasound (MRgFUS). However, given the high costs related to MR imaging, an alternative monitoring modality combining real-time capabilities with lower costs would be preferred.

Temperature increase in tissue results in a change of the sound velocity and to tissue expansion. Both phenomena have an impact on the speckle pattern of the US image. Accordingly, US images can be analyzed for retrieving information on the local temperature change, which potentially enables ultrasound guided focus ultrasound, known as USgFUS.

For developing such an ultrasound-based temperature monitoring, an algorithm that compares speckle patterns of ultrasound B-mode images (obtained through plane wave compounding) generated before and after the heating was implemented. This comparison is realised by a cross-correlation of the A-scans considering some optimization method to calculate physically realistic time shifts between the speckles. The algorithm was implemented in 2D (for linear arrays) and 3D (matrix array data) and initially tested on synthetic phantom data generated in Matlab. Qualitative temperature maps could be obtained when a spherical hotspot of a radius of 2-3 $\mathrm{mm}$ in a depth of 20-30 $\mathrm{mm}$ was assumed.

In a second step, the algorithm was applied to phantom data obtained by local heating inside an US phantom or in chicken breast. Signals were acquired with a multichannel platform (DiPhAS /Fraunhofer IBMT, 128 channels @ 80 MSamples/s digitization) in plane wave compounding mode. Qualitative temperature maps could be retrieved in 2D using a linear array transducer for detection ( $7 \mathrm{MHz}, 128$ elements, LA7, Vermon SA). As a next step, the method is currently expanded to $3 \mathrm{D}$ by using a newly developed matrix array (11 x 11 elements, $2 \mathrm{MHz}$ frequency, by Fraunhofer IBMT). 


\section{Multichannel system for translational research in high intensity focused ultra- sound}

Steffen Tretbar ${ }^{1}$, Marc Fournelle $^{1}$, Christoph Risser ${ }^{1}$, Holger Hewener ${ }^{1}$, Andreas Melzer, ${ }^{2}$

${ }^{1}$ Ultrasound Department, Fraunhofer IBMT, Sankt Ingbert, Germany

${ }^{2}$ Innovation Center Computer Assisted Surgery, Leipzig, Germany

e-mail: steffen.tretbar@ibmt.fraunhofer

In research on different HIFU-applications (neuromodulation, hyperthermia, immunotherapy), fundamental processes are first investigated on cell models and stepwise translated to small animal models and humans. For this reason, we developed a translational ultrasound system for application in different settings from in-vitro to clinical.

This new modular research platform consists of a mainboard accommodating 16 frontboards (16 RX/TX channels each), a PC (connected to the mainboard via PCIe), a power board and a connector board for integration of different ultrasound applicators. The system is upgradable with 1-2 boosters (128 channels) for high-power applications. It works as a standalone system with touch screen based UI and provides open software interfaces to MATLAB, C++, C\# or an API. In addition, the system is MR-compatible. The 256 transmission channels provide up to $+/-100 \mathrm{~V}$ (adjustable) in the frequency range $0.1-20 \mathrm{MHz}$. The tri-state pulsers allow the transmission of square wave burst signals with programmable frequency, number of periods and delays for each channel, however the duty cycle (DC) is limited to $<10 \%$.

In combination with a 128-channel booster, it allows free pulse coding ( $f=0.1-5 \mathrm{MHz}$ ), an output power of up to 16 W/channel @ $50 \bullet$ and a DC up to $100 \%$ for max. 100s. In addition, the 256 receive channels are available for therapy control/imaging. As a conclusion, a new open MR-compatible US research platform has been developed and characterized. The capability to drive different transducers (96-well-applicator, matrix-arrays for small animal/clinical settings) has been demonstrated and enables translational research for many HIFU applications. 


\section{Close Contour Packaging of Printed Circuit Boards Using Additive Manufactur- ing}

Valerie M. K. Werner, Institute of Medical and Polymer Engineering, Technical University of Munich, Garching, Germany, valerie.werner@tum.de

Jonas Frey, Institute of Medical and Polymer Engineering, Technical University of Munich, Garching, Germany, frey.jonas@gmx.de

Stefan Fischer, Institute of Medical and Polymer Engineering, Technical University of Munich, Garching, Germany, st.fischer@tum.de

Markus Eblenkamp, Institute of Medical and Polymer Engineering, Technical University of Munich, Garching, Germany, markus.eblenkamp@tum.de

In medical technology, the use of electronic components for applications of wearables, implantables, and disposable in vitro diagnostics has increased in recent years. Additive manufacturing offers the possibility of individualization, miniaturization, and high degree of functional integration. The aim of this study was the development of a strategy for the close contour integration of a printed circuit board (PCB) equipped with temperature sensors into thermoplastics by means of Fused Layer Modeling (FLM). First, the adhesion of the polymer materials nylon 680, nylon 618, polylactide, and acrylonitrile-butadiene-styrene to the PCB surface (epoxy-based solder resist) was investigated. The standardized pull-off test for adhesion was performed. Influencing factors were identified with regard to printer settings, pretreatment measures for electronic components, and material parameters. Second, an additive packaging process was developed that aimed the integration of miniaturized electronic components (SMD design) into the printed structure the closest possible to the contour. Third, in order to check the thermal load of the SMD components, the temperature sensors were completely printed with the filament and supplied with energy for real-time monitoring during the printing process. A positive influence on the adhesive forces could be determined by increasing the printing bed temperature, tempering the PCB, and plasma pretreatment of the substrate. A combination of parameters could be determined which leads to adhesive strengths of up to $4 \mathrm{GPa}$. A maximum temperature load of $111^{\circ} \mathrm{C}$ on the temperature sensor was measured at a processing temperature of $260^{\circ} \mathrm{C}$ at the nozzle. The thermal stress caused by the process was so low that there was no danger of destruction either of the electrical components or solder joints. The presented process enables the encapsulation of conventionally produced PCBs by means of additive packaging. Thus, a high degree of functional integration and individualized external geometries of medically compatible plastic party can be achieved. 


\section{Towards automated and painless venipuncture - a review on vibratory needle insertion methods}

Jan Liu, Institute of Medical Device Technology, University of Stuttgart, Stuttgart, Germany, jan.liu@imt.uni-stuttgart.de

Kent Stewart, Institute of Medical Device Technology, University of Stuttgart, Stuttgart, Germany, kent.stewart@imt.uni-stuttgart.de

Peter P. Pott, Institute of Medical Device Technology, University of Stuttgart, Stuttgart, Germany, peter.pott@imt.uni-stuttgart.de

The insertion of a needle into soft, in-homogeneous tissue is required for many medical procedures including blood draw, tissue biopsy, drug delivery, and brachytherapy. For blood draw, precise needle placement is critical as a partially punctured or a completely pierced blood vessel will cause bruising or hematoma leading to increased pain and discomfort for the patient. Commonly, the needle insertion is performed manually by a clinician after identifying the target blood vessel and planning an appropriate angle and depth. Limited feedback exists to aid the clinician, with resistance of needle movement and blood flow being the only indicator to clinicians of successful insertion into the vessel. An automated procedure of needle insertion could increase the precision of needle placement by eliminating human error. In addition, different needle insertion methods could be implemented. It has been shown that vibratory needle insertions methods have the ability to reduce frictional forces during insertion, which is in turn correlated with increased needle placement precision. In general, needle bending, tissue deformation, and movement of potential targets away from the needle are also directly proportional to needle insertion forces. Lower insertion forces also result in a reduced frequency and intensity of the pain and trauma felt. Additionally, the injury from insertion has the potential to recover faster. Another approach is to override the pain sensed by applying a dominant sensation of vibration corresponding to the gate control theory of pain. In this paper, different vibratory needle insertion methods that have been studied experimentally are reviewed, addressing tissue characteristics, force measurement, vibration generation, outcomes (force, precision) and limitations. The review concludes with a discussion and a definition of future challenges. 